\title{
An evaluation of six-year Stenotrophomonas maltophilia infections in a university hospital
}

\author{
Nevin Ince ${ }^{1}$, Dilek Yekenkurul ${ }^{1}$, Ayşe Danış², Emel Çalışkan³ ${ }^{3}$ İdris Akkaş ${ }^{4}$
}

1. Department of Infectious Diseases and Clinical Microbiology, Duzce University Faculty of Medicine, Duzce, Turkey.

2. Duzce University Research and Application Hospital, Infection Control Committee Nurse Duzce, Turkey.

3. Department of Clinical Microbiology, Duzce University Faculty of Medicine, Duzce, Turkey.

4. Department of Infectious Diseases, Oltu State Hospital, Ministry of Health, Erzurum, Turkey.

\section{Author details:}

Dilek Yekenkurul Tel: +90 5345271222 e-mail: dr.dilekakinci@gmail.com; Ayşe Danış Tel: +90 5325016396 e-mail: adanis@windowslive.com; Emel Çalışkan Tel: +90 5352640114 e-mail: emelcaliskan81@yahoo.com.tr; İdris Akkaş Tel: +90 5537249484 e-mail: i.dr.is@hotmail.com

\begin{abstract}
Background: Stenotrophomonas maltophilia is a Gram-negative bacillus and opportunistic emergent pathogen causing hospital-acquired infections (HAIs). Due to risk factors such as prolonged intensive care unit stay and invasive procedures, it has become one of the leading causes of HAIs.

Objective: The aim of this study was to evaluate the epidemiology of S.maltophilia infections over a six-year period at Düzce University Hospital, Turkey.

Methods: The incidence, clinical characteristics, antimicrobial susceptibility and outcomes of nosocomial S. maltophilia infections during this period were retrospectively analyzed.

Results: During the study period, 67 samples obtained from 61 patients were identified. Pneumonias $(82 \%)$ were the most common HAIs, followed by bloodstream infections (10.5\%), urinary tract infections (3\%), skin and soft tissue infections $(3 \%)$ and surgical site infection (1.5\%). Admission to intensive care, hospitalization exceeding 30 days, and previous use of broad-spectrum antibiotics constituted risk factors. Resistance to cotrimoxazole $(6 \%)$ was lower than that to levofloxacin $(18 \%)$.

Conclusion: The most important risk factors for S.maltophilia infection in patients are previous exposure to antibiotics, prolonged hospitalization and invasive procedures such as mechanic ventilation. Discharging patients as early as possible with the rational use of antibiotics may be effective in reducing $S$. maltophilia infections and resistance rates.
\end{abstract}

Keywords: Stenotrophomonas maltophilia, hospital-acquired infections, epidemiology, risk factors.

DOI: https://dx.doi.org/10.4314/ahs.v20i3.13

Cite as: Ince N, Yekenkurul D, Danıs A, Calhskean E, Akkaș I. An evaluation of six-year Stenotrophomonas maltophilia infections in a university hospital. Afri Health Sci. 2020;20(3): 1118-1123. https:// dx.doi.org/10.4314/abs.v20i3.13

\section{Introduction}

Stenotrophomonas maltophilia is an aerobic, non-fermentative, motile, sporeless, gram-negative bacillus widely
Corresponding author:
Nevin Ince,
Department of Infectious Diseases
and Clinical Microbiology,
Duzce University Faculty of Medicine,
Duzce, Turkey.
Tel: +905053505839, Fax: +903805421387
e-mail: drnevince@gmail.com

present in nature ${ }^{1}$. It is currently the only species in the genus Stenotrophomonas ${ }^{2}$. The agent can be present in oropharyngeal and airway flora, and can be isolated from several environments in which humans live. It was previously thought to be a pathogen only in immunosuppressive diseases, but is now included among the agents implicated in nosocomial infections due to such risk factors as extended hospitalization, intensive care stay, and invasive procedures, even in immunocompetent patients ${ }^{1,2}$. Although cases have been reported in community-acquired infections, it is frequently isolated from infections developing in hospital ${ }^{3}$. Patients with a high risk of S.maltophilia infection include subjects with 
a previous history of antibiotic therapy, chronic liver or kidney disease, connective tissue disorders, immune suppression due to HIV-positivity or malignancy, who are attached to mechanical ventilators or under follow-up in intensive care units, or with severe underlying comorbid disease ${ }^{4,5}$. Difficulties may be encountered in the treatment of S.maltophilia, which is naturally resistant to several antibiotics, such as penicillins, cephalosporins, carbapenems, and aminoglycosides ${ }^{6}$. Bacterial resistance to drugs develops for reasons such as biofilm formation, synthesis of drug-neutralizing enzymes, synthesis of false targets impervious to the drug, or alteration of permeability against the $\mathrm{drug}^{7,8}$. An increase in resistance rates has been observed to antibiotics such as cotrimoxazole and levofloxacin?.

The epidemiology of nosocomial infections with S.maltophilia varies greatly, depending on the healthcare institution profile and geographical location. Evaluation of local data is therefore essential in order to assess trends over time and to describe the national situation compared to international data. The purpose of this retrospective study was to examine the clinical characteristics, underlying risk factors, and antibiotic resistance rates of nosocomial S. maltophilia infections in 2013-2018, and to compare the results with other findings in the literature.

\section{Methods}

This retrospective study was conducted at the Duzce University Education and Research Hospital, located in the northwest of Turkey and serving as a tertiary care referral hospital, from January 2013 to December 2018. All hospitalized patients aged 18 years or above with nosocomial infections caused by S.maltophilia were included in the study. Patients' medical records of patients, including clinical microbiology and Hospital Infection Control Committee reports, were evaluated, and demographic features, clinical conditions, laboratory data, antimicrobial susceptibility, and outcomes were analyzed retrospectively. Ethics committee approval for our study was obtained from the Düzce University Faculty of Medicine Ethics Committee (No. 2019/127). Nosocomial infections were diagnosed based on Centers for Disease Control and Prevention (CDC) recommendations ${ }^{10}$. Since nosocomial S.maltophilia infection developed twice at different times in six of the 61 patients, the sample number was calculated as 67 . The patient number ( $\mathrm{n}: 61)$ was therefore used in calculations involving patient age, sex, unit of admission, risk factors, and mortality rates (n:61), while S.maltophilia numbers (n:67) were used in calculations such as specimen type, infection diagnosis, and antibiotic sensitivity.

\section{Laboratory methods}

Clinical samples sent to the microbiology laboratory under appropriate conditions were added to $5 \%$ sheep blood agar and Eosin Methylene Blue (EMB) (Oxoid, UK) agar and incubated in an aerobic environment for $24 \mathrm{~h}$ at $35-37^{\circ} \mathrm{C}$. Blood specimens placed into blood culture tubes under sterile conditions for culture were incubated without delay in a BACTEC blood culture device. Five percent sheep blood agar and EMB agar was added to tubes with positive growth. Conventional methods and a Vitek-2 automated system (BioMérieux, France) were used to identify the growing bacteria and to determine their antbiotic sensitivities. Bacterial antibiotic sensitivities were determined in the light of Clinical Laboratory Standards Institute (CLSI) recommendations in 2013-2014, and 2015-2018 European Committee on Antimicrobial Susceptibility Testing (EUCAST) recommendations in 2015-2018.

\section{Statistical analysis}

Statistical analysis was performed on SPSS for Windows 14.0 software. The descriptive values of the data obtained were expressed as mean, standard deviation, number, and percentage frequencies.

\section{Results}

During the six-year study period, 67 samples from $61 \mathrm{pa}-$ tients were identified as culture-positive for S.maltophilia. Sixty-six percent (n:40) of the patients were men, and the patients' mean age was $64.34 \pm 19.24$ years (1893). Mean length of hospitalization was $75.01 \pm 58.02$ days, with $82 \%$ (n:50) of patients admitted to the intensive care unit and 18\% (n:11) admitted to the wards. In terms of admission diagnoses at initial hospitalization, $33 \%$ (n:22) were admitted with a preliminary diagnosis of infection. Eighty-two percent (n:55) of the nosocomial infections developing with S.maltophilia consisted of pneumonia (n:55). Other diagnoses included bacteremia $(10.5 \%)$, urinary tract infection $(3 \%)$, cutaneous and soft tissue infections (3\%) and surgical site infections $(1.5 \%)$. Use of mechanical ventilation was present as an invasive procedure in two-thirds of patients (72\%). The other most commonly identified risk factors were admission to intensive care ( $82 \%)$, hospital stay exceeding 30 days $(74 \%$, and previous use of broad-spectrum antibiotics (64\%). The general mortality rate in all patients with S.maltophilia growth was 49\% (n:30). Demographic data for patients with nosocomial infection and patient characteristics are shown in Table 1. 
Table 1: Demographic and basic characteristics of the 61 patients infected with $S$. maltophilia

\begin{tabular}{|c|c|}
\hline Patient characteristics & n (\%) \\
\hline Age (mean) & $64.34 \pm 19.24(18-93)$ \\
\hline \multicolumn{2}{|l|}{ Sex } \\
\hline Male/Female & $40 / 21$ \\
\hline Days of hospitalization (mean \pm SD) & $75.01 \pm 58.02$ \\
\hline \multicolumn{2}{|l|}{ Admission Diagnosis } \\
\hline Infectious diseases & $22(33)$ \\
\hline Cerebrovascular disease & $13(19.5)$ \\
\hline Respiratory insufficiency & $8(12)$ \\
\hline Trauma & $7(10.5)$ \\
\hline Cardiac arrest & $6(9)$ \\
\hline Other & $11(16)$ \\
\hline \multicolumn{2}{|l|}{ Comorbid Diseases } \\
\hline Hypertension & $28(61)$ \\
\hline $\mathrm{DM}$ & $16(26)$ \\
\hline Heart failure & $12(20)$ \\
\hline Cerebrovascular disease & $10(16)$ \\
\hline COPD & $8(13)$ \\
\hline Malignancy & $7(11)$ \\
\hline Chronic kidney failure & $4(7)$ \\
\hline Other & $28(46)$ \\
\hline \multicolumn{2}{|l|}{ Nosocomial Infection Diagnosis } \\
\hline Pneumonia & $55(82)$ \\
\hline Bacteremia & $7(10.5)$ \\
\hline Urinary tract infection & $2(3)$ \\
\hline Skin and soft tissue infection & $2(3)$ \\
\hline Surgical site infection & $1(1.5)$ \\
\hline \multicolumn{2}{|l|}{ Devices Used } \\
\hline Invasive mechanical ventilation & $44(72)$ \\
\hline Central venous catheter & $28(46)$ \\
\hline Urinary catheter & $58(95)$ \\
\hline \multicolumn{2}{|l|}{ Other risk factors } \\
\hline Follow-up in intensive care & $50(82)$ \\
\hline Surgical intervention & $14(23)$ \\
\hline Broad-spectrum antibiotic use & $39(64)$ \\
\hline Hospitalization exceeding 30 days & $45(74)$ \\
\hline Mortality & $30(49)$ \\
\hline
\end{tabular}

Analysis of isolated strains by years revealed an increase in 2015-2017, but a decrease in 2018. Tracheal aspirate constituted $75 \%$ (n:50) of the specimens collected. The others were collected from phlegm in four, catheter in four, blood in three, urine in two, and bronchoalveolar lavage in one. Forty seven of the 67 S.maltophilia infected patients had co-infection. The most common agent in polymicrobial samples was Acinetobacter baumannii. All 67 isolates were tested for antimicrobial resistance, and the most susceptible antibiotics were levofloxacin and cotrimoxazole $(82 \%$ and $94 \%$ susceptibility, respectively). No strains were simultaneously resistant to both antibiotics. Distributions of samples by years and specimen type antibiotic sensitivities are shown in Table 2. 
Table 2: Strain distributions by years, specimen characteristics, and antibiotic susceptibilities

\begin{tabular}{|c|c|}
\hline \multicolumn{1}{|c|}{ Sample characteristics } & n (percentage) \\
\hline Year & \\
2013 & $5(7.5)$ \\
2014 & $5(7.5)$ \\
2015 & $20(30)$ \\
2016 & $19(28.5)$ \\
2017 & $13(19)$ \\
2018 & $5(7.5)$ \\
& \\
Specimen type & $50(75)$ \\
Deep tracheal aspirate & $4(6)$ \\
Phlegm & $4(6)$ \\
Catheter & $3(4)$ \\
Blood & $3(4)$ \\
Wound & $2(3)$ \\
Urine & $1(1.5)$ \\
Bronchoalveolar lavage & \\
Antibiotic susceptibility & \\
Levofloxacin & $55(82)$ \\
Cotrimoxazole & $63(94)$ \\
& \\
\hline
\end{tabular}

\section{Discussion}

Increasing numbers of nosocomial infections developing due to S.maltophilia are being reported for reasons such as prolonged hospitalization, the use of broad-spectrum antibiotics, and an increase in the numbers of immunosuppressive patients ${ }^{11}$. The most common infections developing with this bacterium are pneumonia and bloodstream infections, while wound and urinary tract infections are less common ${ }^{12}$. The current retrospective study aimed to identify the differences of demographic and clinical characteristics, microbiological findings and the final mortality outcomes of patients with nosocomial infection caused by $S$. maltophilia.

S.maltophilia is a microorganism that can be found in the hospital environment. It causes colonization in various medical devices, leading to nosocomial infection ${ }^{11}$. High rates of polymicrobial infection have also been reported in these patients ${ }^{13}$. A high rate of polymicrobial growth $(70 \%)$ was also determined in the present study. The most common agent in polymicrobial specimens was Acinetobacter spp. Candevir Ulu et al. also identified Acinetobacter spp. as the most frequently seen agent $(40.1 \%)^{14}$. We attribute this to low hand hygiene compliance in intensive care units at that time. Studies in which S.maltophilia has particularly been isolated in airway and blood specimens have also determined this most commonly in airway specimens $(75 \%)$.
Due to the greater prevalence of various predisposing factors, patients admitted to intensive care represent the majority of patients in studies involving S.maltophilia ${ }^{15}$. Intensive care patients constituted $82 \%$ ( $\mathrm{n}: 50$ ) of the patients in our study. Other risk factors for S.maltophil$i a$ in our study were hospitalization exceeding 30 days (74\%), use of broad-spectrum antibiotics (64\%), and mechanical ventilation (72\%). Previous studies have reported mortality rates of $12-69 \%$ for S.maltophilia infection $^{4,8,15}$. In agreement with the previous literature, the mortality rate in our study was $49 \%$. The inconsistent findings may be due to variations in patient populations or in other factors contributing to mortality. A history of use of broad-spectrum antibiotics prior to infections developing with S.maltophilia has been reported as a risk factor in several previous studies ${ }^{4}$. Previous treatment with antipseudomonal broad-spectrum antibiotics has been reported as a risk factor for S.maltophilia bacteremia in some studies ${ }^{16}$. In contrast, Sumida et al. reported that previous use of antibiotics effective against methicillin-resistant Staphylococcus aureus, but not carbapenems, represented a risk factor for S.maltophilia bacteremia ${ }^{17}$. The rate of previous antibiotic use in our study was $64 \%$, and the fact that antibiotic groups were not differentiated is one of the limitations of this study. The fact that antibiotics such as carbapenem and cephalosporin that are frequently employed in empiric treatment are naturally resistant to $S$. maltophilia delays treatment until cultures are obtained and increases mor- 
tality ${ }^{18}$. One recent review study determined an increase in rates of $S$. maltophilia resistance to cotrimoxazole and levofloxacin. According to that review study, susceptibility dtermined in different countries was $80-99 \%$ for TMP-SXT and $44-97 \%$ for levofloxacin ${ }^{19}$. One study of two centers in Turkey at different times reported a time-dependent decrease in cotrimoxazole susceptibili$t^{20,21}$. The low cotrimoxazole resistance in our study is a welcome finding for our institution as a local datum. Since $S$. maltophilia is generally susceptible to quinolones, these have become an important empiric treatment option on non-fermentative bacterial infections ${ }^{22}$. Among susceptibility studies involving levofloxacin, Gozel et al. from Turkey determined a low level of $S$. maltophilia levofloxacin resistance, at $2.9 \%{ }^{21}$. In contrast, Cho et al. reported that their high rate of resistance to levofloxacin, $56 \%$, derived from their patient group consisting of individuals with hematological malignan$\mathrm{cy}^{23}$. The levofloxacin resistance rate in our study was $18 \%$, which is in agreement with the previous literature. The use of levofloxacin in our hospital may need to be evaluated to achieve further improvement in levofloxacin susceptibility.

\section{Conclusion}

There are a number of limitations to this study. The first is its retrospective nature. Other important limitations include the sample size, which might be insufficient for adequately assessing the prognostic factors in S. maltophilia-associated infection. Further epidemiological multi-center studies involving longer surveillance are therefore now needed for a better understanding of the prevalence and distribution of $S$. maltophilia-associated nosocomial infections.

\section{Source of support}

None.

\section{Conflict of interest}

None declared.

\section{References}

1. Senol E. Stenotrophomonas maltophilia: the significance and role as a nosocomial pathogen. J Hosp Infect. 2004; $57: 1-7$

2. Low CY, Rotstein C. Emerging fungal infections in immunocompromised patients. F1000 Rep. 2011; 3:14. 3. Mooney L, Kerr KG, Denton M. Survival of Stenotrophomonas maltophilia following exposure to concentrations of tobramycin used in aerosolized therapy for cystic fibrosis patients. Int J Antimicrob Agents. 2001; 17:63-6.

4. Xun M, Zhang Y, Li BL, Wu M, Zong Y, Yin YM. Clinical characteristics and risk factors of infections caused by Stenotrophomonas maltophilia in a hospital in Northwest China. J Infect Dev Ctries. 2014; 8:1000-5.

5. Naeem T, Absar M, Somily AM. Antibiotic resistance among clinical isolates of Stenotrophomonas maltophilia at a teaching hospital in Riyadh, Saudi Arabia. J Ayub Med Coll Abbottabad. 2012; 24:30-3.

6. Valdezate S, Vindel A, Loza E, Baquero F, Cantón R. Antimicrobial susceptibilities of unique Stenotrophomonas maltophilia clinical strains. Antimicrob Agents Chemother. 2001; 45: 1581-4.

7. Dagata EM. Antimicrobial resistant, gram positive bacteria among patients undergoing chronic hemodialysis. Clin Infect Dis. 2002; 15:1212-18.

8. Wu H, Wang JT, Shiau YR, Wang HY, Lauderdale TL, Chang SC; TSAR Hospitals. A multicenter surveillance of antimicrobial resistance on Stenotrophomonas maltophil$i a$ in Taiwan. J Microbiol Immunol Infect. 2012; 45:120-6.

9. Ç1kman A, Parlak M, Bayram Y, Güdücüoğlu H, Berktaş M. Antibiotics resistance of Stenotrophomonas maltophilia strains isolated from various clinical specimens. Afr Health Sci. 2016; 16:149-52.

10. Garner JS, Jarvis WR, Emori TG, Horan TC, Hughes JM. CDC definitions for nosocomial infections. Am J Infect Control. 1988; 16:128-40.

11. Brooke JS. Stenotrophomonas maltophilia: an emerging global opportunistic pathogen. Clin Microbiol Rev. 2012; 25: 2-41.

12. Dizbay M, Tunçcan ÖG, Maral I, Aktaş F, Şenol E. Five year surveillance of nosocomial Stenotrophomonas maltophilia infections in Gazi University Hospital. Türkiye Klinikleri J Med Sci. 2009; 29:1406-11.

13. Lai CH, Chi CY, Chen HP, et al. Clinicalcharacteristics and prognostic factors of patients with Stenotrophomonas maltophilia bacteremia. J Microbiol Immunol Infect. 2004; 37:350-8.

14. Candevir Ulu A, Kurtaran B, Kibar F, et al. 2007'den 2011'e Stenotrophomonas maltophilia Infeksiyonları. Flora Dergisi. 2013; 18:119-27.

15. Araoka H, Baba M, Yoneyama A. Risk factors for mortality among patients with Stenotrophomonas maltophilia bacteremia in Tokyo, Japan, 1996-2009. Eur J Clin Microbiol Infect Dis. 2010; 29: 605-8.

16. Hotta G, Matsumura Y, Kato K, et al. Risk Factors and Outcomes of Stenotrophomonas maltophilia Bacteraemia: A Comparison with Bacteraemia Caused by Pseudomonas aeruginosa and Acinetobacter Species. PLoS One. 2014;9(11): e112208. 
17. Sumida K, Chong Y, Miyake N, et al. Risk Factors Associated with Stenotrophomonas maltophilia Bacteremia: A Matched Case-Control Study. PLoS One. 2015;10(7):e0133731.

18. Metan G, Uzun O. Impact of initial antimicrobial therapy in patients with bloodstream infections caused by Stenotrophomonas maltophilia. Antimicrob Agents Chemother. 2005; 49:3980-81.

19. Gajdács M, Urbán E. Epidemiological Trends and Resistance Associated with Stenotrophomonas maltophilia Bacteremia: A 10-Year Retrospective Cohort Study in a Tertiary-Care Hospital in Hungary. Diseases. 2019; 31:7. 20. Caylan R, Kaklikkaya N, Aydin K, et al. An epide- miological analysis of Stenotrophomonas maltophilia strains in a university hospital. Jpn J Infect Dis. 2004; 57:37-40.

21. Gozel MG, Celik C, Elaldi N. Stenotrophomonas maltophilia Infections in Adults: Primary Bacteremia and Pneumonia. Jundishapur J Microbiol. 2015; 8(8): e23569. 22. Weiss K, Restieri C, De Carolis E et al. Comparative activity of new quinolones against 326 clinical isolates of Stenotrophomonas maltophilia. I Antimicrob Chemother. 2000; 45:363-5.

23. Cho SY, Lee DG, Choi SM, et al. Stenotrophomonas maltophilia bloodstream infection in patients with hematologic malignancies: A retrospective study and in vitro activities of antimicrobial combinations. BMC Infect Dis. 2015; 15:69. 